\title{
El universo de la novela de Denis Diderot
}

\section{Claudia RUIZ GARCÍA \\ Universidad Nacional Autónoma de México}

\begin{abstract}
Este artículo explora cuatro novelas o antinovelas de Denis Diderot - La Religieuse, Les Bijoux indiscrets, Le neveu de Rameau y Jacques le fataliste- que permiten conocer algunos aspectos de la visión del mundo del filósofo así como su particular concepción sobre este género. Se revisa la aceptación o el rechazo de esta parte de su producción en su tiempo así como las razones de la censura para condenarla.
\end{abstract}

Palabras ClaVE: Diderot, novela, La Religieuse, Les Bijoux indiscrets, Le neveu de Rameau y Jacques le fataliste.

This article explores four novels or antinovels written by Denis Diderot $-L a$ Religieuse, Les Bijoux indiscrets, Le neveu de Rameau y Jacques le fatalistewhich enable the knowledge of some aspects of the philosopher's worldview as well as his particular conception about this genre. The acceptance or rejection of this part of his production during his time is revised, as well as the reasons of the censorship to condemn it.

KeY words: Diderot, novel, La Religieuse, Les Bijoux indiscrets, Le neveu de Rameau y Jacques le fataliste.

\author{
Imposez-moi silence sur la religion et le gouvernement, \\ et je n'aurai plus rien à dire. \\ Diderot, La Promenade du sceptique.
}

Este año toca hacer un reconocimiento por los trescientos años de su nacimiento a una de las figuras emblemáticas de la Modernidad y del Siglo de las Luces: Denis Diderot.

Se trata de una personalidad que incursiona en todos los terrenos del pensamiento de su tiempo: filósofo materialista, pensador político, teórico de arte y literario, pionero de la educación laica y de Estado, codirector de la Enciclopedia, máximo monumento de la epistemología ilustrada y novelista. Sería imposible abordar cada una de estas facetas, sin embargo, me gustaría, a través de un acercamiento a su producción como 
autor de novelas y relatos cortos, desentrañar algunas de las claves de su compleja visión del mundo. Dentro de este rubro, Diderot escribe y publica alrededor de quince textos de gran interés para el estudio de la novela moderna. Junto con su producción filosófica es ésta una de las más prolíficas. Inicia su carrera como traductor y editor y estas dos disciplinas lo conducirán más tarde al terreno de la filosofía y la literatura. Es también el camino que recorren muchas personalidades del siglo XVIII, quienes mantendrán lazos muy estrechos entre estos dos campos del conocimiento. Este filósofo, a la par que Voltaire, Montesquieu, Rousseau y otros tantos, escriben novelas y cuentos filosóficos, atendiendo a lo que Sartre más tarde resumirá en su elocuente frase que señala que "une tecnique romanesque renvoie toujours à la métaphysique du romancier" (Sartre: 71). De esta forma entendemos la postura de uno de los estudiosos de la obra diderotiana, Laurent Versini, quien, ampliándola a otros dominios del saber, afirma: "Son esthétique et son écriture, sa politique et sa morale reposent sur sa métaphysique. Aucune œuvre forte n'existe sans poser les éternelles questions de Dieu, du mal, du sexe et de la Cité" (Versini: 2).

Así, su obra narrativa responde a estas preguntas, lo que explica las razones por las que parte de ella no pudo publicarse en su tiempo. Su carácter subversivo obligó al autor a esconderla y ha sido únicamente en el siglo XX y la primera década del XXI, gracias a la apertura del fondo familiar y de las colecciones de la zarina, Catalina la Grande, en San Petesburgo (Versini: 2), como se han rescatado algunos manuscritos, permitiendo de esta forma restituir las versiones originales y corregir las desfiguradas, como fue el caso de su antinovela Jacques le fataliste, así como sacar a la luz textos desconocidos durante más de dos siglos como el de su relato Mystification.

Hasta hoy en día se le atribuyen Les bijoux indiscrets, L'oiseau blanc, conte bleu, La Religieuse, Mystification, La vision de M. de Bignicourt, Les deux amis de Bourbonne, Entretien entre un père avec ses enfants, Ceci n'est pas un conte, Madame de la Carlière, Supplément au voyage de Bougainville, Première satire, Lui et moi, Le neveu de Rameau y Jacques le fataliste. Como se puede observar, la propuesta es enorme y variada aunque un tanto desigual, lo que marcó, en alguna medida, la aceptación o el rechazo de sus textos en la posteridad. Brunetière, por ejemplo, en el siglo XIX lo clasificó como "Diffus, désordonné, confus, débraillé, grossier, cynique, barbare en art, inepte en religion, vicieux en morale" (Versini: 3). No obstante, a mediados del siglo XX la percepción de esta parte de su obra cambió radicalmente, pues se le consideró como un precursor de la revolución narrativa de esa época, en la que podrían reconocerse novelistas como Gide y los miembros de "Le Nouveau Roman". Pero la obra de Diderot no sólo se conforma de relatos que cuestionen o pongan en duda el género novelesco, pues si bien Diderot a menudo advierte a su lector, señalando que tal narración no es una novela como en Jacques le fataliste o en su relato Ceci n'est pas un conte, también escribe textos que obedecen a las convenciones narrativas del siglo XVIII, donde cabrían, por ejemplo, La Religieuse y Les bijoux indiscrets.

Con este último grupo me gustaría comenzar. En primer lugar, por La Religieuse, pues es una de las novelas más importantes de su repertorio y una de las más bellas. Esta 
obra Diderot la presenta para que sea difundida por entregas en la Correspondance littéraire, un periódico manuscrito destinado a un círculo reducido de lectores selectos de Europa (Martin: 13), con objeto de realizar "la plus effrayante satire des couvents" (apud. Martin: 190). Interesa ver de cerca el propósito de Diderot, sobre todo por el tipo de repercusiones religiosas, políticas y económicas que éste tendría en su momento, pues el convento es una de las piezas clave de la educación en la configuración del Antiguo Régimen. El tema de la clausura importa a varios sectores de la sociedad y esta frase traduce bien el proyecto de Diderot que persigue sacudir una institución primordial del catolicismo, cuestionada a lo largo de su historia. Desde tiempos de la Reforma se escuchan las voces de personalidades de la talla de Erasmo de Rotterdam y de Martín Lutero, posteriormente la de los pensadores libertinos del siglo XVII, como Cyrano de Bergerac, y finalmente la de los filósofos del Siglo de las Luces, como sería, por ejemplo, Montesquieu. Para los primeros, el convento o monasterio se reduce a ser una cárcel para solteras. Erasmo de Rotterdam, por ejemplo, en uno de sus Coloquios afirma:

\begin{abstract}
Je maudis ceux qui attirent des adolescents ou de jeunes filles [...] en profitant de leur naïveté ou de leur superstition pour leur faire croire qu'il n'y a pas d'espoir de salut hors des monastères [...]. Si un jour je suis obligé d'exprimer ce que je pense sur ce sujet, je peindrai ces faiseurs d'esclaves et la grandeur de leur forfait de telle sorte qu'il n'y aura personne pour nier que j'ai eu quelque raison de donner cet avertissement, d'ailleurs en termes civils, pour ne pas donner aux méchants une occasion de faire mal (697).
\end{abstract}

Para los eruditos libertinos, la castidad, uno de los votos que hacen las novicias en estos centros de reclusión, es totalmente antinatural. Cyrano de Bergerac en su utopía planetaria Voyage dans la Lune integra un diálogo donde el narrador-protagonista discute este tema con uno de sus interlocutores selenitas y este último le dice:

Qu'on ne m'objecte point les beaux panégyriques que la virginité, cet honneur n'est qu'une fumée, car enfin tous ces respects dont le vulgaire l'idolâtre ne sont rien, même entre vous autres, que de conseil, mais de ne pas tuer, mais de ne pas faire son fils, en ne faisant point, plus malheureux qu'un mort, c'est le commandement. Pourquoi je m'étonne fort, vu que la continence du monde d'où vous venez est tenue si préférable à la propagation charnelle, pourquoi Dieu ne vous a pas fait naître à la rosée du mois de mai comme les champignons, ou, tout au moins, comme les crocodiles du limon gras de la terre échauffé par le soleil. Cependant il n'envoie point chez vous d'eunuques que par accident, il n'arrache point les génitoires à vos moines, à vos prêtres, ni à vos cardinaux (87).

Esto mismo opina Montesquieu en sus Lettres Persanes. En la carta CXVII que Usbek escribe a Rhédi, al opinar sobre esta práctica, le dice:

La prohibition du divorce n'est pas la seule cause de la dépopulation des pays chrétiens: le grand nombre d'eunuques (refiriéndose a los hombres consagrados a la iglesia) qu'ils 


\section{$84 \square$ EL UNIVERSO DE LA NOVELA DE DENIS DIDEROT}

ont parmi eux n'en est pas une moins considérable. Je parle des prêtres et des dervis, de l'un et de l'autre sexe, qui se vouent à une continence éternelle: c'est, chez les chrétiens, la vertu par excellence; en quoi je ne les comprends pas, ne sachant ce que c'est qu'une vertu dont il ne résulte rien. Je trouve que leurs docteurs se contredisent manifestement, quand ils disent que le mariage est saint, et que le célibat, qui lui est opposé, l'est encore davantage; sans compter qu'en fait de préceptes et de dogmes fondamentaux, le bien est toujours le mieux. Le nombre de ces gens faisant profession de célibat est prodigieux. Les pères y condamnaient autrefois les enfants dès le berceau: aujourd'hui, ils s'y vouent eux-mêmes dès l'âge de quatorze ans; ce qui revient à peu près à la même chose. Ce métier de continence a anéanti plus d'hommes que les pestes et les guerres les plus sanglantes n'ont jamais faits. On voit, dans chaque maison religieuse, une famille éternelle, où il ne naît personne, et qui s'entretient aux dépens de toutes les autres. Ces maisons sont toujours ouvertes, comme autant de gouffres ou s'ensevelissent les races futures (265).

Además, en opinión de este filósofo el claustro es un espacio para forjar monstruos y energúmenos. Así, en sus Lettres Persanes utiliza la imagen del serrallo para denunciar los nefastos efectos del encierro. Se sabe que Montesquieu hace, al pintar de forma feroz el modo de aprisionamiento de la mujer en Oriente, un paralelismo con lo que sucede en estos lugares en el mundo de la cristiandad. Diderot conoce de cerca esta problemática pues su hermana enloqueció en el convento de las ursulinas donde fue recluida y allí murió a los veintisiete años. Raymond Trousson señala que para el filósofo:

Le couvent est encore le lieu de tous les désordres mentaux y physiologiques manifestés sous une forme ou une autre: Mme de Moni, qui communique avec l'esprit divin, est une hystérique à tendance mystique, sœur Sainte Christine vit avec fanatisme et sadisme sa névrose personnelle, et c'est la vie claustrale qui rend compte du saphisme de la supérieure de Saint-Eutrope; comme l'esprit, le corps se dérègle: frustration, mélancolie, hystérie, mysticisme, cruauté, folie, saphisme sont le produit de l'outrage fait à la nature (162).

Así vemos a la protagonista de la novela, Suzanne Simonin, recluida y obligada a hacer sus votos contra su voluntad, confrontarse a este tipo de religiosas y padecer las consecuencias de los trastornos producidos por el aislamiento. Al cumplir diecisiete años es incitada por su confesor a tomar el hábito. Ella rechaza la propuesta, sin embargo, sus padres deciden enviarla al convento contra su voluntad. Esta reacción hace despertar una serie de sospechas en la joven y poco a poco logra imaginar la suerte que le depara: aceptar su reclusión a perpetuidad. De antemano está consciente de que no goza del derecho a la dote para casarse, pues tiene dos hermanas mayores que ella, además se pregunta si su padre ha tomado tal decisión para castigar a la madre que cometió adulterio. Así asume — aunque con cierta reserva — su futuro próximo: expiar dicha falta encerrada detrás de los muros de un claustro. A lo largo de las diferentes etapas en su estancia monacal, conviviendo al lado de mujeres perturbadas por los estragos de la reclusión, llega a la siguiente conclusión: 
L'homme est né pour la société. Séparez-le, isolez-le, ses idées se désuniront, son caractère se tournera, mille affections ridicules s'élèveront dans son cœur, des pensées extravagantes germeront dans son esprit, comme les ronces dans une terre sauvage. Placez un homme dans une forêt, il y deviendra féroce; dans un cloître, où l'idée de nécessité se joint à celle de servitude, c'est pis encore; on sort d'une forêt; on ne sort plus d'un cloître; on est libre dans la forêt, on est esclave dans le cloître. Il faut peutêtre plus de force d'âme encore pour résister à la solitude qu'à la misère; la misère avilit, la retraite déprave (Diderot, 1972: 198).

Uno de los aspectos más interesantes de esta novela es que se inscribe dentro del repertorio de relatos del Siglo de las Luces e incluso desde antes, donde el tema del claustro y del encierro obligado aparece de manera recurrente. Desde la literatura medieval y renacentista existe una tradición literaria antimonástica. En ella, la figura de los curas y monjes lascivos, madres superioras lujuriosas y novicias ingenuas invaden los relatos que pretenden ridiculizar a la institución claustral, construyendo una intriga a partir de las diferentes violaciones a un código de conducta que se precia de aplicarse de forma rígida e intachable en estos supuestos recintos sagrados. Allí, al abrigo de la mirada de los otros, se da libre curso a las pulsiones humanas. Se aplica la famosa frase de Cremonini que dice: "A l'intérieur fait comme il te plait, à l'extérieur agis selon la coutume" (apud, Martin: 26). Pero también se denuncia un orden social que condena al encierro a jóvenes que constituyen una amenaza para la integridad y preservación del patrimonio familiar. El convento y el monasterio son los basureros donde van a parar los hijos que, por su posición en orden de nacimiento, se convierten en una carga o desecho.

Otra de las convenciones literarias utilizadas por Diderot consiste en recurrir a la moda de las ficciones inscritas en un marco de exotismo, muy en boga en ese momento. En Les bijoux indiscrets el espacio geográfico correspondería a lo que hoy en día se conoce como el Congo. Versini considera que la astucia de esta novela exótica reside en la intención de hacer una caricatura política y social, pues el personaje principal, Mangogul, es Luis XV; Mirzoza es Madame de Pompadour, la favorita del rey, y la corte de Banza, Versalles, con todo un código de etiqueta y galantería que raya en el ridículo. ${ }^{1}$ Mangogul se describe con rasgos muy similares a los de la personalidad de Luis $\mathrm{XV}$, como su propensión al tedio y a la favorita por su inclinación a la protección de escritores de la época. Esta estrategia, nos dice Versini, ya había sido utilizada por Crebillon hijo en un texto titulado Zeokunizul roi des Konfinans (anagrama de "Louis roi des français") (Versini: 21). Se trata de una novela poco uniforme donde Diderot acumula anécdotas picantes, retratos atrevidos, disertaciones estéticas o filosóficas, que

\footnotetext{
${ }^{1}$ Un ejemplo es la moda entre las damas de honor de poseer perritos. El sultán pregunta a su favorita: “je vous assure que je vois des chiens à toutes les femmes de ma cour, et que vous m'obligerez de m'apprendre pourquoi elles en ont, ou pourquoi vous n'en avez point. La plupart d'entre elles en ont même plusieurs; et il n'y en a pas une qui ne prodigue au sien des caresses qu'elle semble n'accorder qu'avec peine à son amant. Par où ces bêtes méritent-elles la préférence?” (Diderot, 1981: 135).
} 


\section{$86 \square$ EL UNIVERSO DE LA NOVELA DE DENIS DIDEROT}

le valieron uno de sus encarcelamientos en la prisión de Vincennes (Rustin: 8). Diderot aclara que la génesis de este texto está en un hecho totalmente anodino:

J'ai écrit un livre abominable: Les bijoux indiscrets. Je pourrais en partie m'en excuser: J'avais une maîtresse. Elle me demanda cinquante louis d'or, et je n'avais pas un sou. Elle menaça de me quitter si je ne pouvais pas lui donner cette somme au bout de la quinzaine. Je rédigeai alors le livre conformément au goût du plus grand nombre de nos lecteurs. Je l'apportai chez le libraire, il me compta les cinquante louis d'or, et je les jetai dans la jupe de ma belle (Rustin: 9-10).

En este relato, el sultán recibe de un genio un anillo mágico que tiene el don de hacer hablar las partes genitales (les bijoux) de las mujeres. Diderot no inventa nada nuevo pues en la tradición medieval esta convención literaria existe en el célebre fabliau del Chevalier qui fist parler les cons. Sin embargo, deslindándose de la misoginia del cuento popular, resulta interesante la actitud del sultán en el relato del filósofo. Allí se ilustra la falta de virtud y de honestidad de las mujeres, y aunque el retrato de ellas no es nada halagüeño, ya que se les presenta propensas al vicio, en varias ocasiones el discurso del rey deja entrever su reconocimiento a su difícil situación. La mujer aparece como víctima de una enajenación que la somete a un orden arbitrario o tiránico, lo que la lleva a utilizar la hipocresía y las perversiones secretas como sus únicos recursos para sobrevivir. Así lo afirma: “J'ai prétendu [...], que les femmes sages étaient rares; et loin de me médire, j'ajouterais volontiers qu'il est surprenant [en esas condiciones] qu'elles ne le soient pas davantage" (Diderot, 1981: 63).

Dice Nicolas Rousseau (50) que a Les Bijoux indiscrets se le consideró por mucho tiempo como una novela de entretenimiento y muy menor respecto al resto de su obra, pues se le relacionó únicamente por algunos capítulos que abordaban cuestiones filosóficas. No obstante, hoy en día ha generado un gran interés pues ilustra ampliamente la validez de sus teorías sobre la física experimental. Para este estudioso es evidente que:

Quand le sultan expérimente le pouvoir de son anneau magique sur le bijou des femmes, c'est peut-être par plaisir ou par curiosité, mais ses essais n'en servent pas moins à renseigner sur le fondement réel des passions humaines. Difficile également de ne pas voir que toutes les observations ainsi dégagées, Mirzoza les résume dans sa métaphysique (dite aussi expérimentale), laquelle lui fait notamment affirmer que l'âme s'est par nature placée dans le corps de l'homme 'comme dans un vaste palais dont elle n'occupe pas toujours le plus bel appartement' (51).

Se entiende entonces la importancia que adquiere este anillo porque hace posible escuchar la verdadera voz de la naturaleza o de la sexualidad, tema tabú en el contexto del filósofo. Estas dos novelas - La Religieuse y Les Bijoux indiscrets-demuestran de manera elocuente de qué forma Diderot se afirma como novelista peligroso y contestatario en su siglo por las temáticas que aborda. Pero, si bien sorprende por la posición que asume contra la moral y las prácticas dominantes de su tiempo, también consterna porque cuestiona de manera radical el género novelesco. 
Dentro del repertorio de textos que se reconocen en este grupo, el novelista recurre con frecuencia a la estrategia de no caer en la tentación de construir una historia o intriga de manera lineal y que obedezca a cierta lógica. A menudo rechaza la supuesta omnisciencia del escritor sobre sus personajes y, dentro del cuerpo de los relatos, inserta argumentos que sirven para destruir y denunciar algunas convenciones narrativas. De allí que algunos de éstos lleven en el título o en el subtítulo el término "sátira" que, atendiendo a uno de sus sentidos, designa a cualquier obra conformada por un conjunto heterogéneo o por una mezcla de muchas cosas, a la manera de un popurrí y que persigue a la vez ridiculizar las costumbres o los hombres de su tiempo. En este grupo de novelas y cuentos podemos incluir Le Neveu de Rameau, Jacques le fataliste, Ceci $n$ 'est pas un conte, Mystification y otros más. Veamos los dos primeros textos ya que ilustran claramente la posición de Diderot como uno de los críticos más agudos de los propios alcances de la novela.

Apegándose a un criterio meramente cronológico, habría que hablar primero de $L e$ Neveu de Rameau, aunque haya pocos y confusos indicios sobre la posible fecha de su publicación. Referencias dispersas del propio Diderot permiten intuir que la redacción de esta obra la inició en 1762 y la concluyó en 1774, aunque en Francia solamente se editó hasta 1891 y antes de 1805 circuló una traducción al alemán de Goethe (Delon: 11-12). En ella el autor confronta dos voces: Lui/Él, es decir; el músico fracasado, sobrino del gran Jean-Philippe Rameau y Moi/Yo, el filósofo. El diálogo se interrumpe por pasajes narrados que probablemente vienen del "Moi/Yo" e incluyen comentarios y reflexiones sobre los sentimientos que su interlocutor le provoca. Este diálogo, sin orden ni concierto, permite confrontar dos puntos de vista sobre opiniones muy personales respecto al arte, la moral, la educación y la existencia. Un tema se encadena con el otro y si en un principio quien lleva la batuta de la conversación es "Moi/Yo", es decir el filósofo, cuya lucidez y superioridad interroga y juzga al otro, poco a poco los roles se invierten. Al final es el sobrino de Rameau quien dirige la discusión, acorralando o poniendo en aprietos al filósofo, erigiéndose en un pensador de la pérdida de los valores (Delon: 17). Así él le dice al filósofo que cree que éste se ha interesado en su persona, a pesar de ser un pobre diablo y un cínico, porque su charla lo divierte (Diderot, 2006: 59). Más tarde, hablando sobre la felicidad, el honor y la virtud le señalará que conoce a mucha gente honesta que no es feliz, mientras que hay muchos que sí lo son sin ser para nada honestos (86), y agregará que todo mundo alaba a la virtud, pero todos en realidad la detestan y huyen de ella por ser glacial en un mundo donde hay que tener los pies bien calientes (87). En otro momento el filósofo le pregunta si alguna vez, al sustraer algo que no le pertenecía, resintió algún remordimiento y éste le contesta:

Ho, sans remords. On dit que si un voleur vole l'autre, le diable s'en rit [...] Dans la nature, toutes les espèces se dévorent; toutes les conditions se dévorent dans la société. Nous faisons justice les uns des autres, sans que la loi s'en mêle. La Deschamps autrefois; aujourd'hui la Guimard [cortesanas que coleccionaban amantes poderosos] 


\section{$88 \square$ EL UNIVERSO DE LA NOVELA DE DENIS DIDEROT}

venge le prince du financier; et c'est la marchande de mode, le bijoutier, le tapissier, la lingère, l'escroc, la femme de chambre, le cuisinier, le bourrelier, qui vengent le financier de la Deschamps. Au milieu de tout cela, il n'y a que l'imbécile ou l'oisif qui soit lésé, sans n'avoir vexé personne; et c'est fort bien fait. D'où vous voyez que ces exceptions à la conscience générale, ou ces idiotismes moraux dont on fait tant de bruit, sous la dénomination de tours de bâton ne sont rien; et qu'à tout, il n'y a que le coup d'œil qu'il faut avoir juste (80-81).

Esta obra tan singular no recibió ningún comentario favorable en vida del autor. Incluso Diderot no hizo referencia alguna al Neveu en su correspondencia. Probablemente este silencio se deba al temor del filósofo de que alguno de sus contemporáneos se sintiera aludido por las opiniones vertidas ya fuera por uno u otro de los interlocutores de este diálogo. Una suerte muy parecida a ésta la tuvo Jacques le fataliste, novela nada convencional, que desconcertó a los lectores de su siglo. En este relato Diderot multiplica las digresiones, las interrupciones de la historia principal (si es que puede decirse que hay una), las anécdotas, los relatos intercalados y las miradas en retrospectiva. Al igual que en el Neveu de Rameau, el diálogo ocupa un lugar y una función primordial. Dos personajes, Jacques y su amo, viajan sin conocer el destino final de su trayecto ni por cuanto tiempo. Desde las primeras líneas de la novela Diderot prepara al lector a enfrentarse a una obra donde el autor se niega a situarlo en una posición cómoda, es decir, dentro de lo que sería una verdadera ficción. La novela inicia así: "Comment s'étaient-ils rencontrés? —Par hasard comme tout le monde. —Comment s'appelaient-ils? —Que vous importe? —D'où venaient-ils? —Du lieu le plus prochain. - Où allaient-ils? —Est-ce que l'on sait où l'on va? — Que disaient-ils? -Le maître ne disait rien; et Jacques disait que tout ce qui nous arrive de bien et de mal ici-bas était écrit là-haut"’2 (Diderot, 1970: 25).

Estos dos personajes viajan y a lo largo de su recorrido charlan sobre cualquier tema, se detienen en hostales, a la manera de don Quijote y Sancho, entablan conversaciones con la gente que encuentran, cuentan o escuchan historias. A veces la voz de un narrador los interrumpe y se dirige a su lector, haciéndolo interactuar con lo que sucede en el relato pues lo invita a ser testigo o a responder a las preguntas que le plantea. Se anuncia que Jacques hablará sobre sus amores pero este relato, del que no se conocerá el desenlace, se interrumpirá por las historias del marqués de Arcis y de Madame de la Pommeraye, la historia del caballero de Saint-Ouin y la del padre Hud-

\footnotetext{
${ }^{2}$ Jacques encontrará siempre situaciones cómicas para explicar cómo el destino ya está preestablecido. Por ejemplo, en uno de los diferentes diálogos que mantiene con su amo le dira: "Et qui est-ce qui a fait le grand rouleau où tout est écrit? Un capitaine, ami de mon capitaine, aurait bien donné un petit écu pour le savoir; lui, n'aurait pas donné une obole, ni moi non plus; car à quoi cela me servirait-il? En éviterais-je pour cela le trou où je dois m'aller casser le cou?" Su amo le responde que tal vez sí y Jacques replica: "Moi je crois que non; car il faudrait qu'il y eût une ligne fausse sur le grand rouleau qui contient la vérité, qui ne contient que vérité, et qui contient toute vérité. Il serait écrit sur le grand rouleau: Jacques se cassera le cou tel jour', et Jacques ne se cassera pas le cou? Concevez-vous que cela se puisse quel que soit l'auteur du grand rouleau?" (Diderot, 1970: 35).
} 
son. Trousson considera que: "L'apparent désordre d'un roman où le romancier se refuse à jouer les démiurges et prétend ignorer ce que feront ses personnages, reproduit celui du monde réel. Diderot peut donc dire et répéter qu'il ne fait un roman, puisqu'il dédaigne les procédés qu'un romancier ne manquerait pas d'employer. Tout ce qu'il y a de sûr, c'est qu'il faut accepter la nécessité universelle" (292).

Versini, como Trousson al analizar la forma deshilvanada de esta novela, sin saber realmente si detrás de este intento de antinovela se persigue tocar un tema tan serio como el de la fatalidad, llega a la siguiente conclusión: hay una incompatibilidad al menos aparente entre la discontinuidad, el relato gratuito y la necesidad de un encadenamiento inexorable que el fatalismo pretende descubrir en el universo. Sin embargo, no hay ninguna certeza de esto, pues el relato está impregnado de ambigüedades. No pretende ser una novela y al menos los tres relatos referidos anteriormente sí obedecen a las convenciones de este género en su tiempo. Por ello podemos concluir reconociendo, como lo hace Paul Vernière que: "Diderot dans cet étrange roman tient sans doute à nous amuser, mais son propos permanent, comme dans toute son œuvre, est d'exprimer l'essentielle ambiguïté de notre condition et de notre vocation morale. Si, à l'aide d'une technique déroutante, il nous introduit dans l'univers arbitraire de Jacques, c'est pour nous rappeler que c'est univers est le nôtre" (Vernière: 17).

Como si ese desorden que impera a lo largo del supuesto viaje de Jacques y su amo, incoherente y absurdo en apariencia, fuera un espejo de lo que sucede en la realidad. Esto justificaría la presencia de esa voz narrativa que interviene constantemente para burlarse de las estrategias utilizadas por los novelistas para crear quimeras. Tal voz rompe de manera reiterada la ilusión de la ficción, lo que produce una insatisfacción en el lector, quien termina por desinteresarse por la historia principal y por preguntarse si realmente existe un hilo conductor del relato o lo que importa es fijar la atención en el diálogo entre Jacques y su amo y así desentrañar una filosofía.

Dice Françoise Barguillet (247) que Diderot guardó algunos manuscritos de sus novelas sin apresurarse a publicarlos. Según esta especialista, la razón de esta actitud podría ser la prudencia, pero también la vergüenza y cierta aprensión al juicio de su tiempo que pudiera considerarlo un novelista. En efecto, en sus escritos teóricos sobre arte y literatura la novela no ocupa un lugar primordial si se le compara a su inclinación por la música y la pintura. Salvo algunos autores de su tiempo como Richardson, Sterne, Marivaux y el abad Prévost, ${ }^{3}$ no hay indicios que muestren un marcado interés por las grandes novelas de su siglo. Incluso reconoce sus limitaciones y las expresa en la primera frase del discurso preliminar de su obra La promenade du sceptique ou les

\footnotetext{
${ }^{3}$ Sin embargo en Jacques le fataliste la voz del narrador que interpela a cada momento al lector le dice a éste: "la vérité, la vérité! - La vérité, me direz-vous, est souvent froide, commune et plate; par exemple, votre dernier récit du pansement de Jacques est vrai, mais qu'y a-t-il d'intéressant? Rien. —D'accord. —S'il faut être vrai, c'est comme Molière, Regnard, Richardson, Sedaine; la vérité a ses côtés piquants, qu'on saisit quand on a du génie; mais quand on en manque? Quand on en manque, il ne faut pas écrire" (Diderot, 1970: 59).
} 
allées donde dice: "Les prétendus connaisseurs en fait de style chercheront en vain à me déchiffrer. Je n'ai point de rang parmi les écrivains connus. Le hasard m'a mis la plume à la main; et trop de dégoûts accompagnent la condition d'auteur, pour que dans la suite je me fasse une habitude d'écrire" (apud. Artigas-Menant: 13).

Qué equivocado estaba Diderot, pues si bien su siglo y el siguiente no valoraron su producción novelística, después ese vacío crítico se llenó de estudios que posicionaron a este autor como una pieza clave en la historia de la novela francesa y de la novela moderna.

\section{Obras citadas}

Artigas-Menant, Geneviève. 2011. "Introduction”. La lettre clandestine: Diderot et la littérature clandestine. Revue annuelle publiée avec le concours du CNRS et de la région Rhône-Alpes. París: Presses Universitaires Paris-Sorbonne.

BArguillet, Françoise. 1981. Le roman au XVIII siècle. París: Presses Universitaires de France.

Bergerac, Cyrano. 1970. Voyage dans la lune. Ed. Maurice LaugGa. París: Garnier-Flammarion.

Diderot, Denis. 2006. Le Neveu de Rameau. Ed. Michel Delon. París. Gallimard. 1994. Euvres, t. II. Ed. Laurent Versini. París. Robert Laffont.

1981. Les Bijoux indiscrets. Ed. Jacques Rustin. París: Gallimard.

1972. La Religieuse. Ed. Robert MAUZI. París: Gallimard.

1970. Jacques le fataliste. Ed. Paul Vernière. París: Garnier-Flammarion.

ÉrASME. 1991. Euvres choisies. Ed. Jacques ChORMARAT. París: Librairie Générale Française.

Fabiaux et contes des poètes françois des XI, XII, XIII, XIV et XV siècles, tirés des meilleurs auteurs. 1976. Ed. Etienne BARBAZAN. Ginebra: Slatkine Reprints. Martin, Christophe. 2010. La Religieuse de Diderot. París. Gallimard.

Montesquieu. 1973. Lettres persanes. Ed. Jean StAROBINSKI. París: Gallimard.

Rousseau, Nicolas. 1997. Diderot. L'écriture romanesque à l'épreuve du sensible.

París: Honoré Champion.

SARTRE, Jean-Paul. 1947. Situations I. París: Gallimard.

Trousson, Raymond. 2007. Diderot. París: Gallimard. 